\title{
Estrogen modulates cutaneous wound healing by downregulating macrophage migration inhibitory factor
}

\author{
Gillian S. Ashcroft, ${ }^{1}$ Stuart J. Mills, ${ }^{1}$ KeJian Lei, ${ }^{2}$ Linda Gibbons, ${ }^{1}$ Moon-Jin Jeong, ${ }^{2}$ \\ Marisu Taniguchi, ${ }^{3}$ Matthew Burow, ${ }^{4}$ Michael A. Horan, ${ }^{1}$ Sharon M. Wahl, ${ }^{2}$ \\ and Toshinori Nakayama ${ }^{3}$
}

${ }^{1}$ School of Biological Sciences, University of Manchester, Manchester, United Kingdom

${ }^{2}$ Oral Infection and Immunity Branch, National Institute of Dental and Craniofacial Research,

National Institutes of Health, Bethesda, Maryland, USA

${ }^{3}$ Departments of Medical Immunology and Molecular Immunology, Graduate School of Medicine,

Chiba University, Chiba, Japan

${ }^{4}$ Tulane Medical School, New Orleans, Louisiana, USA

\begin{abstract}
Characteristic of both chronic wounds and acute wounds that fail to heal are excessive leukocytosis and reduced matrix deposition. Estrogen is a major regulator of wound repair that can reverse agerelated impaired wound healing in human and animal models, characterized by a dampened inflammatory response and increased matrix deposited at the wound site. Macrophage migration inhibitory factor (MIF) is a candidate proinflammatory cytokine involved in the hormonal regulation of inflammation. We demonstrate that MIF is upregulated in a distinct spatial and temporal pattern during wound healing and its expression is markedly elevated in wounds of estrogen-deficient mice as compared with intact animals. Wound-healing studies in mice rendered null for the MIF gene have demonstrated that in the absence of MIF, the excessive inflammation and delayed-healing phenotype associated with reduced estrogen is reversed. Moreover, in vitro assays have shown a striking estrogenmediated decrease in MIF production by activated murine macrophages, a process involving the estrogen receptor. We suggest that estrogen inhibits the local inflammatory response by downregulating MIF, suggesting a specific target for future therapeutic intervention in impaired wound-healing states.

J. Clin. Invest. 111:1309-1318 (2003). doi:10.1172/JCI200316288.
\end{abstract}

\section{Introduction}

Because of a complex interaction of medical and epidemiological factors, there has been a rapid increase in the size of the elderly population. With this, there has also been a parallel increase in morbidity associated with age-related delayed wound healing, which costs the health services over $\$ 9$ billion per year. Cutaneous wound healing is characterized by an initial inflammatory response, followed by reformation of the epithelial barrier and matrix deposition. Accumulating evidence implicates inflammation, and the resultant proteolysis of matrix, as a causative factor in age-related delayed healing and suggests that in the absence of infection, the inflammatory response is inappropriately excessive

Received for publication June 26,2002, and accepted in revised form February 6, 2003.

Address correspondence to: Gillian S Ashcroft, School of Biological Sciences, University of Manchester, 3.239 Stopford Building, Oxford Road, Manchester M13 9PT, United Kingdom. Phone: 011-44-(0)161-275 5673; Fax: 011-44-(0)161-275-3915;

E-mail: gillian.s.ashcroft@man.ac.uk.

Conflict of interest: The authors have declared that no conflict of interest exists.

Nonstandard abbreviations used: macrophage migration inhibitory factor (MIF); hormone-replacement therapy (HRT); dehydroepiandrosterone (DHEA); ovariectomization (OVX); recombinant human MIF (rhMIF); estrogen receptor (ER); 2,2-bis-(p-hydroxyphenyl)-1,1,1-trichloroethane (HPTE); propyl pyrazole triol (PPT).
$(1,2)$. There is striking evidence from animal studies dating back to 1962 that estrogens play a crucial role in cutaneous wound healing and repair is significantly delayed in its absence, an event characterized by profound leukocyte recruitment during the initial stages of injury and tissue destruction (3-6). Moreover, exogenous estrogen replacement in these animal and human models reverses the effects on healing (6-8). In this regard, recent reports have shown that hormonereplacement therapy (HRT) prevents the development of chronic wounds (both pressure ulcers and venous ulcers) in postmenopausal women $(9,10)$. Intriguingly, the positive effect of topical estrogen has been shown in both elderly women and men, reflecting the marked decrease in local estrogenic activity secondary to reduced ovarian activity in women, a decline in the adrenal estrogenic precursor dehydroepiandrosterone (DHEA) in men and women, and altered aromatization of systemic precursors to local active estrogen (6).

In excessive and chronic inflammatory disorders, the influx of leukocytes occurs unabated, leading to enhanced cytokine and chemokine production, further unchecked leukocyte recruitment, and ultimately proteolytic tissue destruction. Estrogen accelerates the cutaneous wound-healing process, associated with enhanced matrix deposition, rapid epithelialization, and a dampening of the inflammatory response (2-8). One potential downstream target for hormonal effects 
is macrophage migration inhibitory factor (MIF). MIF is expressed in human endometrium and has been implicated in numerous reproductive responses, including ovulation, blastocyst implantation, and embryogenesis $(11,12)$. MIF is a protein consisting of 115 amino acid residues that has emerged as an important regulator of inflammation, influencing both the innate and antigen-specific functions of the immune system $(13,14)$. First described as a T cell-derived 12 -kDa protein, MIF has recently been shown to be produced by a variety of cell types, including monocytes, endothelial cells, keratinocytes, and anterior pituitary cells, suggesting multifunctional physiological effects (15-18). Beyond its proinflammatory roles in lymphocyte activation and nitric oxide synthesis, MIF acts as a counter-regulator of the anti-inflammatory effects of glucocorticoids, leading to increased monocyte production of IL- 6 , IL-1 $\beta$, and IL-8 (19). The spectrum of proinflammatory activities has recently been extended to direct effects on cytokine expression; MIF induces the production of, and is induced by, TNF- $\alpha(19,20)$. Moreover, MIF can be induced by a variety of inflammatory mediators, including LPS and IFN- $\gamma$, leading to unchecked inflammation (20). Recent studies have implicated MIF in both acute and chronic inflammatory conditions, including antigen-induced arthritis and renal disease; however its role as a modulator in wound repair has not been determined $(21,22)$. To this end, we have documented the role of MIF in an impaired model of cutaneous wound healing and have shown that in the absence of estrogen, MIF underlies the altered in vivo inflammatory response and aberrant wound healing. Systemic and topical estrogen can reverse age-related impaired healing in animal models and in elderly humans. We postulate that one mechanism for such action involves MIF, and inhibiting MIF is a focused therapeutic strategy to potentially accelerate age-related impaired healing states.

\section{Methods}

Wound-healing experiments. Eight- to ten-week-old female mice (MIF BALB/C wild-type and MIF null) (23) were anesthetized with inhaled isofluorane, and the dorsum was shaved and cleaned with alcohol. In addition, 10week-old female mice that had undergone ovariectomization (OVX) 1 month previously were used for the wound-healing experiments. Two equidistant $1-\mathrm{cm}$ fullthickness incisional wounds, placed $1 \mathrm{~cm}$ from the base of the skull and $1 \mathrm{~cm}$ from either side of the midline, were made through the skin and panniculus carnosus muscle and left to heal by secondary intention (i.e., the wound edges were not closed by sutures). The wound sections were bisected and processed so that the midpoint of the wound was sectioned and compared between groups. Wounds were harvested at days 3, 7, and 14 after wounding and were bisected for histology and for RNA analysis and protein extraction (snap frozen in liquid nitrogen). For a subgroup of mice, before wounding, skin was injected intradermally at the site to be incised with $200 \mathrm{ng}$ per wound of TGF- $\beta 1$ (24) (R\&D Systems, Minneapolis, Minnesota, USA), $500 \mathrm{ng}$ per wound of recombinant human MIF (rhMIF) (R\&D Systems), or PBS vehicle, and wounds were excised at day 3 or day 7. The one-dose intradermal injection of $100 \mu \mathrm{l}$ was placed $1 \mathrm{~cm}$ from the skull base and $1 \mathrm{~cm}$ lateral to the midline, and the area was incised immediately after injection. A dose-response study was used to investigate the effects of neutralizing anti-rat MIF antibodies at doses of $500 \mathrm{ng}, 1 \mu \mathrm{g}$, or $2 \mu \mathrm{g}$ per wound (25). In addition, a subgroup of mice, at the time of OVX, underwent estrogen replacement at physiological doses using 0.05-mg slow-release pellets (Innovative Research of America, Sarasota, Florida, USA) inserted subcutaneously. Cardiac puncture was performed on all animals, and blood was processed for serum extraction. In vivo wound gapes were measured immediately after wounding (midpoint of the $1-\mathrm{cm}$ incision) and at daily intervals until wound harvest. At days 3, 5, and 7 after wounding, the mechanical properties of wounds in a subgroup of animals were determined using a BTC1000 system (SRLI Systems, Nashville, Tennessee, USA), which utilizes a vacuum to induce wound breakage and measures wound-breaking strength. These time points were chosen because they consistently induced wound breakage (at day 14 after wounding, the majority of wounds fail to break in all mice).

Histology, immunocytochemistry, and image analysis. Histological sections were prepared from wound tissue fixed in $10 \%$ buffered formalin and embedded in paraffin. Five-micrometer sections were stained with hematoxylin and eosin or subjected to immunohistochemistry with antibodies to human MIF $(0.05 \mu \mathrm{g} / \mathrm{ml} ; \mathrm{AB}$ 289, R\&D Systems), Mac3 (rat anti-Mac3, Pharmingen, San Diego, California, USA), TNF- $\alpha$ (rabbit antimouse, Genzyme Pharmaceuticals, Cambridge, Massachusetts, USA), vWf (rabbit anti-human, DAKO, Glostrup, Denmark), or collagen I (Chemicon, Chandlers Ford, Hampshire, United Kingdom) used at a dilution of 1:20 in PBS (vWF used at 1:25). Human peripheral-blood circulating mononuclear leukocytes were isolated using a Ficoll gradient and resuspended to $5 \times 10^{6}$ cells per milliliter in PBS, and $20 \mu \mathrm{l}$ was transferred to a Vectabond-coated slide. Cells were allowed to adhere for 10 minutes at room temperature in a humidified chamber and fixed with $2 \%$ formalin for 20 minutes at $4^{\circ} \mathrm{C}$. Cells were permeabilized using $0.1 \%$ saponin (Sigma-Aldrich, Poole, United Kingdom) and stained with CD14 cyanin anti-human antibody (1:100, Pharmingen) followed by antibodies to estrogen receptor (ER) $\alpha$ (1:10; F10, Santa Cruz) and $\beta$ receptor (26) (sheep anti-human raised to hinge domain, 1: 200; a gift from Phillipa Saunders, MRC Reproductive Unit, Edinburgh, United Kingdom). Primary antibody was detected either using a TRITC or FITC-labeled secondary antibody (1:40 dilution for 20 minutes) or using the VECTOR peroxidase kit as indicated (Vector Laboratories Inc., Burlingame, California, USA). Control slides stained with each secondary antibody in isolation and 
each primary antibody with the inappropriate secondary were negative. Image analysis and quantification of cell numbers per unit area $\left(\mathrm{mm}^{2}\right)$, wound area, and staining intensity were performed using an Image Pro Plus program (MediaCybernetics Inc., Silver Springs, Maryland, USA). Wound areas were determined by manually drawing the wound margins below the epidermis or clot, down the edge of the wound margin (which can easily be seen where thick collagen bundles appear as representative of intact skin), and above a line drawn between the two edges of the panniculus carnosus muscle. Blinded scoring of histological slides was undertaken, where 0 indicated normal skin-staining intensity, as previously described (2). We used a scale of -5 to +5 (where 0 indicated normal intact skin, +5 indicated maximal intensity, and -5 indicated minimal intensity), which reduces interobserver variability to a minimum $(\mathrm{SD}=0.5)$. Cell numbers were determined by analyzing four defined areas within the wound below the epidermis and calculating the mean number per unit area. Blood-vessel area was quantified as a percentage of total wound area.

ELISAs and Western blots. Peritoneal macrophages were isolated from female wild-type mice, MIF null mice, and ER- $\alpha$ null mice (Harlan Laboratories, Indianapolis, Indiana, USA) by intraperitoneal lavage with ice-cold sterile PBS and pooled for subsequent studies. Cells were plated at $10^{6}$ cells per milliliter with $500 \mu \mathrm{l}$ per well in serum-free phenol red-free medium, treated with or without LPS $(1 \mu \mathrm{g} / \mathrm{ml})$ for 6 hours, and washed with PBS, and estrogen was added for the subsequent 12 hours $\left(10^{-6}\right.$ to $10^{-11} \mathrm{M}$ estradiol). The ELISA was performed using a coating antibody (monoclonal antiMIF MAB289, R\&D Systems) and a biotinylated antihuman MIF capture antibody (goat anti-human, BAF289, R\&D Systems).

For Western blotting, protein was extracted from murine wound tissue and normal skin using a detergent buffer, and $20 \mu \mathrm{g}$ of protein was used for the blot as previously described (27). Rabbit anti-MIF antibody was used at $1 \mu \mathrm{g} / \mathrm{ml}$, and rabbit anti-TNF- $\alpha$ was used at 2 $\mu \mathrm{g} / \mathrm{ml}$ (Genzyme Pharmaceuticals) with an overnight incubation, followed by detection using the AP kit according to the manufacturer's instructions (Bio-Rad Laboratories Inc, Hertfordshire, United Kingdom).

RNase protection assays. Total RNA was isolated from frozen wound tissue using Trizol. RNase protection assays were carried out on RNA according to the manufacturer's instructions (Pharmingen). Band densities were normalized to the L32 housekeeping gene (for RNase protection assays) using an image analysis program (Image Pro Plus, MediaCybernetics).

MIF promoter reporter construct. A 1-kbp fragment was derived from the MIF promoter (GenBank accession number AF033192) using 129/SvJ ES DNA as a template. The primers used were CAGAACTGGTAGTCCCCA (sense) and TGGACCGGAGGACGGTAC (antisense). The fragment containing 67 to -1029 bp of the MIF gene $5^{\prime}$ flank sequence was cloned into the pGL3 basic plasmid
(Promega Corp., Madison, Wisconsin, USA) with luciferase as a reporter gene. MCF7 (ER-positive) cells were transfected with $5 \mu \mathrm{g}$ of DNA per $60-\mathrm{mm}$ plate using Superfect (Qiagen, Cologne, Germany). pGL3 basic vector alone was used as a negative control, pGL3 control vector alone was used as a positive control, and transfection efficiency was determined by $\beta$-galactosidase vector (Promega Corp.). After 48 hours of incubation, cells were treated with or without LPS $(1 \mu \mathrm{g} / \mathrm{ml})$, estrogen $\left(10^{-6} \mathrm{M}\right.$ to $\left.10^{-10} \mathrm{M}\right)$ with and without propyl pyrazole triol (PPT) $\left(10^{-8} \mathrm{M}\right), 2,2$-bis-(p-hydroxyphenyl)-1,1,1-trichloroethane (HPTE) $\left(10^{-7} \mathrm{M}\right)$, or ICI $182,780(100 \mathrm{nM})$ for 12 hours in serum-free media (28-30). Cell lysates were assayed for luciferase or $\beta$-galactosidase activity according to the manufacturer's instructions (Promega Corp.).

Statistical analysis. Statistical differences were determined using the $t$ test or an ANOVA. For data not following a normal distribution, the Mann-Whitney $\mathrm{U}$ test was used. A $P$ value of less than 0.05 was considered significant.

\section{Results}

MIF expression during normal wound healing. Assessment of the temporal profile and cellular sources of MIF expression during normal wound healing in wild-type BALB/C mice, after full-thickness incisional wounds were excised at days 3-14 after wounding, revealed a distinct pattern of expression. Although not apparent in normal epidermis, MIF staining was detected in the suprabasal layers in the migrating epidermis, as has
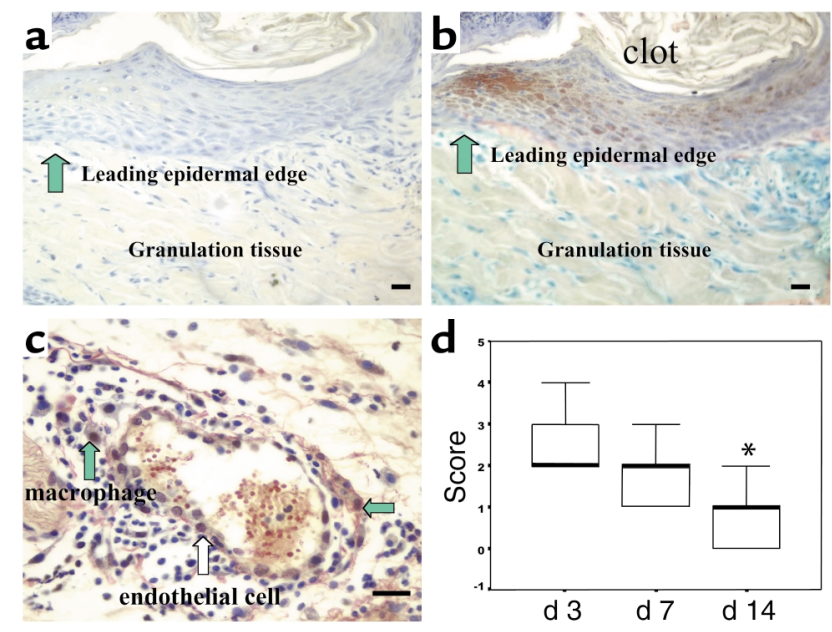

\section{Figure 1}

MIF localizes to keratinocytes, endothelium, and inflammatory cells during wound healing. (a-c) Day 3 wounds from BALB/C mice illustrate suprabasal keratinocyte staining for $\operatorname{MIF}(\mathbf{b}$, arrow), endothelial cells (c, arrow), and perivascular inflammatory cell staining (c, green arrows). (a) represents IgG isotype control negative staining (arrow indicates epidermis). All scale bars represent $10 \mu \mathrm{m}$. (d) Temporal decrease in wound section immunostaining scores for MIF as compared with normal skin (normal skin scored as 0 ). Results represent medians (black lines across boxes), boxes represent interquartile ranges, and the bars extending from the boxes indicate the highest and lowest values ( $n=6$ for each group, ${ }^{*} P<0.05$ versus day 3 ). 
a

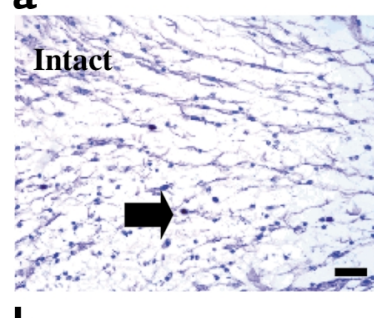

b

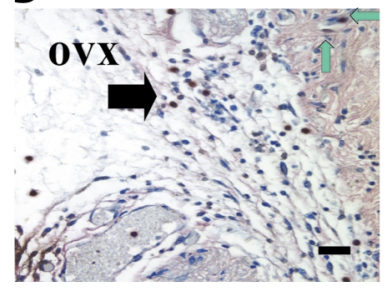

c

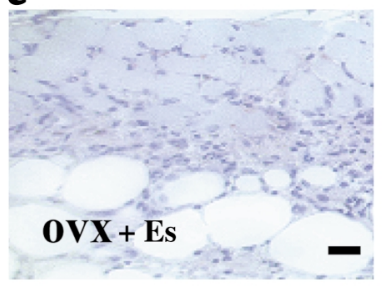

d

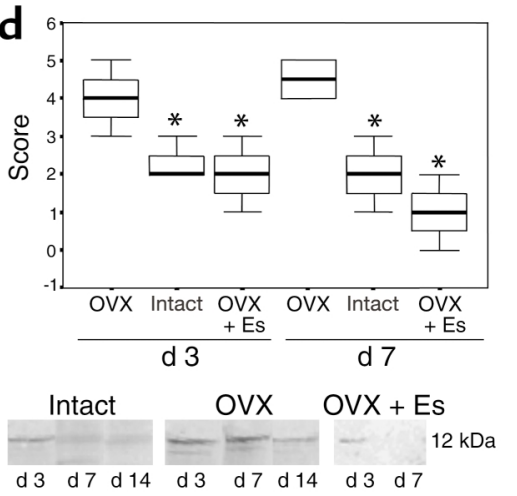

e
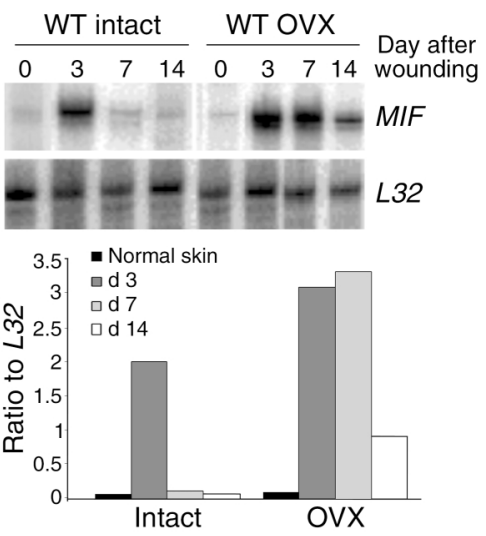

\section{Figure 2}

Reduced circulating estrogen leads to enhanced local MIF expression. (a-c) Inflammatory cells and endothelium stained for MIF at day 7 after wounding (b, green arrows indicate endothelial cells). Inflammatory cell staining (arrows) for MIF was increased in day-7 wounds of OVX mice (b) as compared with their intact littermates (a). Estrogen (Es) replacement in wild-type OVX mice resulted in a marked reduction in MIF levels (c). All scale bars represent $20 \mu \mathrm{m}$. (d) MIF protein levels increased at day 3 (as compared with normal skin, which is scored as 0 ) and declined by day 14 in the wild-type mice (Western blot, lower panel), with significantly increased levels from days 7-14 in the OVX mice. Results represent medians (black lines across boxes), boxes represent interquartile ranges, and the bars extending from the boxes indicate the highest and lowest values $(n=4$, $\left.{ }^{*} P<0.05\right)$. Estrogen replacement in OVX mice reversed this increase in MIF. This temporal pattern was confirmed by Western blotting (d, lower panel). By RNase protection assay, MIF mRNA expression peaked at day 3 (e) and had decreased to basal levels by day 14 after wounding in wildtype intact mice. By contrast, MIF expression was more strongly upregulated from days 3-14 after wounding in the wild-type OVX mice. Normal skin is scored as 0 , and the housekeeping gene is denoted by $L 32$. For both RNA and protein analyses, four mice per time point were used. been reported previously in rodent skin (16) (Figure 1, $\mathrm{a}$ and $\mathrm{b}$ ). Previous studies have described upregulation of MIF in proinflammatory skin disorders (such as atopic dermatitis) in all epidermal layers (31). The suprabasal staining we observed, both in the intact and OVX mice, suggests that specific spatial regulation of MIF expression occurs in wound repair. Inflammatory cell staining and endothelial cell staining were also evident from day 3 after wounding, persisting until day 14 (Figure 1c). Colocalization studies indicated that the MIF-positive cells were macrophages (data not shown). Consistent with the immunohistochemical pattern of increased wound-staining intensity (as compared with normal skin) at day 3 , declining through days 7-14 (Figure 1d), MIF mRNA levels were upregulated at day 3 after wounding and decreased to baseline by day 14 (Figure 2e, wild-type intact). The marked temporal increase in MIF expression during early wound healing implicated a role for this peptide in inflammation and/or repair.

Estrogen modulates MIF expression in vivo. Previous studies have documented that the absence of circulating estrogen results in excessive inflammation, reduced matrix deposition, and delayed wound healing (2). To directly determine the potential contribution of MIF to such impaired wound-healing states, we used mice rendered hypogonadal by OVX. Increased MIF immunostaining was noted from days 3-7 after wounding, localizing to macrophages and endothelial cells (Figure 2, a and $b$ ), with more intense staining in the wounds of the OVX animals than in the intact mice (Figure 2, a and b), which was confirmed by Western blotting (Figure 2d). Replacement of systemic estrogen reduced MIF levels (as compared with OVX mice) as determined by Western blotting and immunohistochemistry (Figure 2, $\mathrm{c}$ and d). A marked increase in wound tissue MIF mRNA expression was observed at days 3 and 7 in the OVX mice as compared with intact mice (Figure 2e). Expression levels had declined by day 14 after wounding in the wounds of the OVX mice but remained greater than those in the intact animals. There were no differences observed in normal-skin MIF expression in the intact and OVX groups. In addition, no differences were noted in MIF serum levels in OVX mice $(740 \mathrm{pg} / \mathrm{ml})$ as compared with intact mice $(820 \mathrm{pg} / \mathrm{ml}, P=0.12)$, suggesting that local regulation of MIF was critical. The upregulation of MIF in the wounds of the OVX mice suggested a direct, or indirect, regulatory role of estrogen on MIF expression.

Effects of MIF deletion on wound healing. We further investigated the role of MIF in the wound-healing process, and its regulation by estrogen, by using mice rendered null for the MIF gene (23). After full-thickness dorsal incisional wounding of intact wild-type and MIF null mice, no significant differences in the rate of healing were observed (Figure 3, a and b). By contrast, the rate of healing in wild-type OVX mice was markedly impaired (Figure 3c). Importantly, initial wound gapes did not differ significantly between all of the groups (wild-type, $1.85 \pm 0.4 \mathrm{~mm}$; wild-type OVX, $1.64 \pm 0.5$ $\mathrm{mm}$; null, $1.7 \pm 0.43 \mathrm{~mm}$; null OVX, $1.68 \pm 0.35 \mathrm{~mm}$ [means $\pm \mathrm{SD}])$. Evident from days 3-7 after wounding, the OVX wild-type animals had enlarged wounds, the 
margins of which failed to close (Figure 3c). Wound healing in the MIF null OVX mice did not differ from that of the null intact mice (Figure 3d). The MIF null OVX group essentially served as a control for both the OVX and the MIF null mice and confirms the hypothesis that if estrogen is regulating expression of MIF, then in the absence of MIF the presence or absence of estrogen is irrelevant. Increased collagen I deposition was observed at day 7 after wounding in the wounds of the OVX null mice as compared with the OVX wildtype mice (Figure 3, e and f). Quantification of crosssectional wound areas from day 3 showed a significant increase in the OVX wild-type mice (Figure $3 \mathrm{~h}$ ) as compared with the OVX MIF null animals. Consistent with the accelerated healing rate and enhanced matrix deposition, the in vivo breaking strengths of the wounds at day 7 after wounding from the OVX MIF null (and intact wild-type) mice were significantly greater than from the OVX wild-type wounds (Figure 3, $i$ and $j$ ).

Effects of MIF deletion on inflammatory mediators. Since both endothelial cells and inflammatory cells predominantly expressed MIF during the wound healing process, we further investigated the consequences of MIF deletion and estrogen deprivation on inflammation and neovascularization. Intriguingly, at day 7 after wounding (Figure 4a), the absence of MIF resulted in a significant decrease in angiogenesis, suggesting that MIF plays an important role in early blood-vessel formation in vivo. Despite previous studies indicating a potential role for estrogen in the angiogenic process, no differences were observed in the area of blood vessels in OVX as compared with intact mice (for both wild-type or MIF null mice) (Figure 4a). Taken together, these data imply that MIF may regulate angiogenesis; however, this does not impede wound healing in the absence of MIF.

In the OVX wild-type animals, not only were the wounds larger, but there was an increase in cells per unit area reflecting a substantial local accumulation of leukocytes (Figure 4, b and c). Histological staining in conjunction with immunostaining using a macrophage-specific antibody indicated that neutrophils and macrophages were significantly increased in the OVX wild-type wounds as compared with the OVX null wounds (Figure 4b). The differences in both wound area and inflammatory responses at day 7 were not significantly different between intact MIF null and wild-type wounds $(P>0.05)$. No differences in circulating peripheral leukocyte counts in wild-type and null mice have
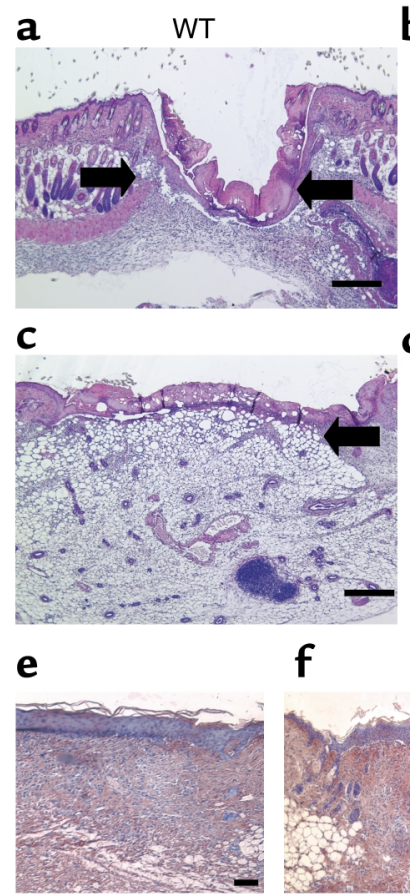

b

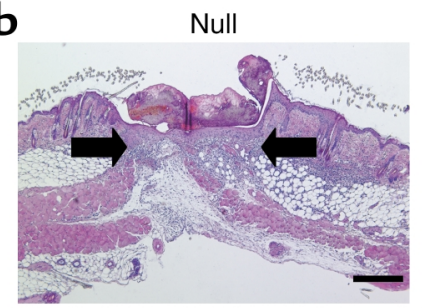

d
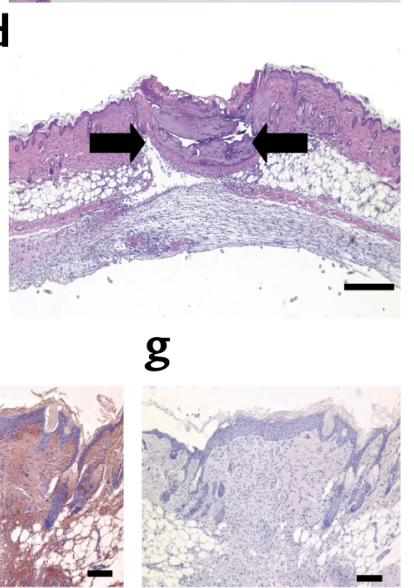

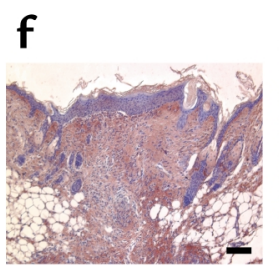

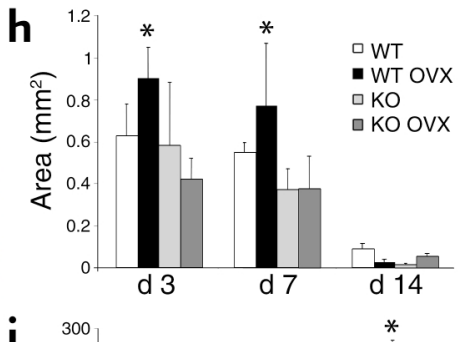
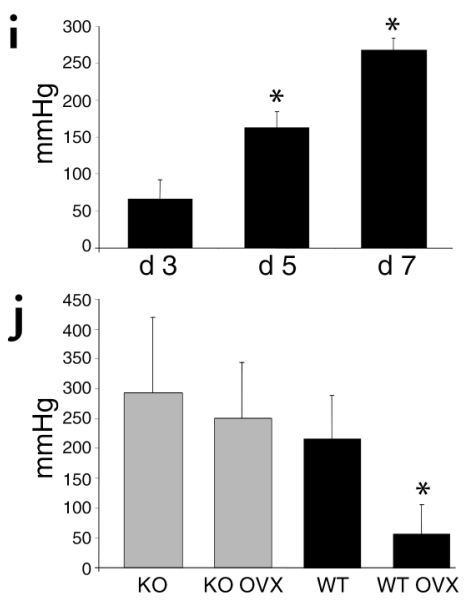

Figure 3

Reduced estrogen has no effect on wound healing in mice null for MIF. Day 3 wounds were wider in OVX wild-type mice (c) with an increased inflammatory response as compared with intact littermates (a). In marked contrast, no differences were observed between wounds from MIF null intact mice (b) and MIF null OVX mice (d). Arrows demarcate wound edges. In (c), the panniculus carnosus muscle is at the extreme edges of the image. Scale bars for a-d represent $100 \mu \mathrm{m}$. In the OVX MIF null wounds at day 7 (f), increased collagen I deposition was observed as compared with wounds from OVX wild-type mice (e). An absence of staining on parallel sections using an isotype control antibody is shown $(\mathbf{g})$. Scale bars for $\mathbf{e}-\mathrm{g}$ represent $10 \mu \mathrm{m}$. Wound areas were significantly greater in OVX wild-type mice than in OVX MIF null mice at day 3 after wounding $(\mathbf{h})$. Results represent means $\pm \operatorname{SEM}\left(n=3-5\right.$ for each group, $\left.{ }^{*} P<0.05\right)$. Wound strength evaluated by disruption of day-3, -5 , and -7 wounds in vivo using a BTC1000 device is shown (i). A significant increase in wound strength was observed at days 5 and 7 as compared with day 3 . Results represent means \pm SEM ( $n=4-5$ for each group, ${ }^{*} P<0.05$ compared to day- 3 data). At day 7 after wounding $(j)$, the ultimate breaking strength $(\mathrm{mmHg})$ was significantly reduced in the OVX wild-type mice as compared with OVX MIF null mice and wild-type intact mice. Results represent means $\pm \operatorname{SEM}\left(n=4,{ }^{*} P<0.05\right)$. 

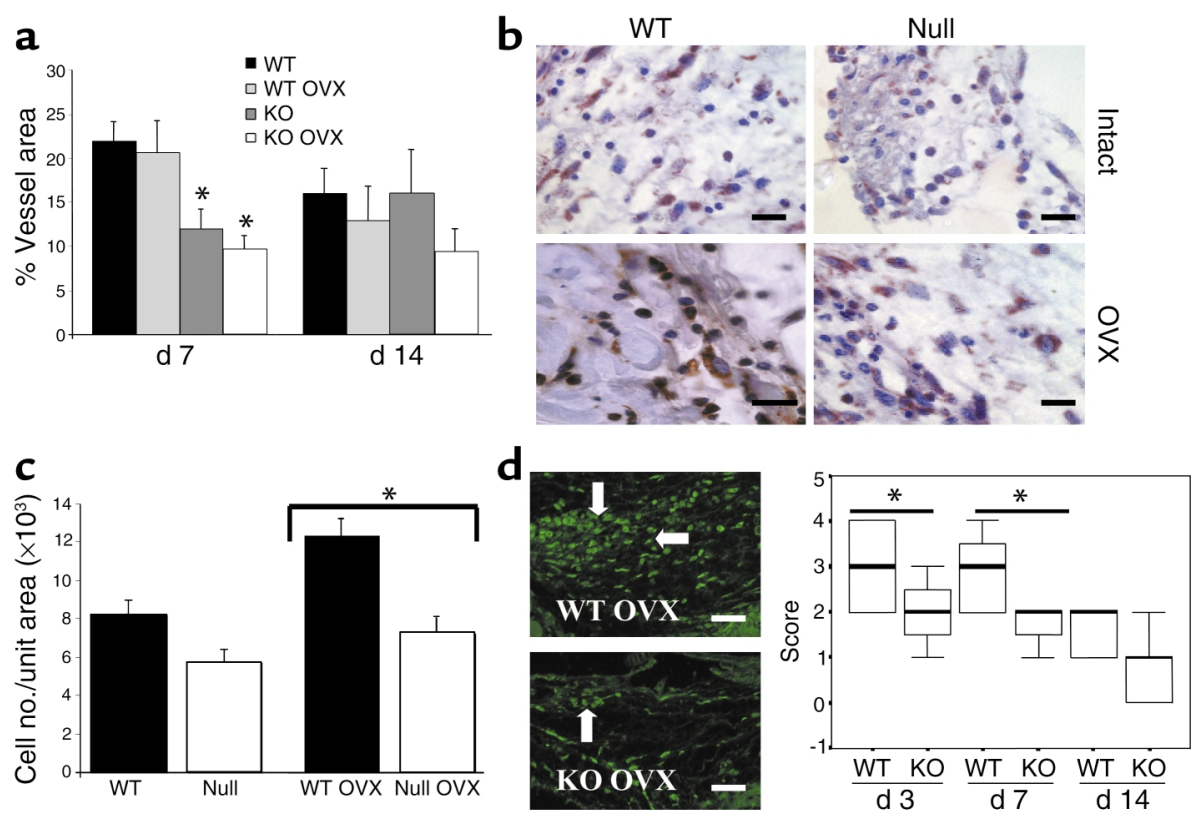

\begin{abstract}
Figure 4
Reduced inflammation in the MIF null wounds. (a) An absence of MIF leads to reduced wound blood-vessel area (vessel area presented as a percentage of total wound area) at day 7; however, an absence of estrogen has no effect on neovascularization. Results represent means $\pm \operatorname{SEM}\left(n=4,{ }^{*} P<0.05\right.$ for comparisons between intact null and intact wild-type or OVX null and OVX wild-type). (b) Mac3 staining illustrates increased inflammatory cell infiltrate in the OVX wild-type wounds as compared with the OVX MIF null wounds at day 3. Scale bars represent $20 \mu \mathrm{m}$. Cell numbers per unit area were quantified at day 3 (c). Results represent means \pm SEM $(n=7-10$ for each group, $\left.{ }^{*} P<0.05\right)$. (d) TNF- $\alpha$ protein levels were increased in the wild-type OVX wounds as compared with the MIF null OVX wounds from days 3-14 after wounding (d, graph) by quantification of immunostaining. Normal skin is scored as 0 . Results represent medians (black lines across boxes), boxes represent interquartile ranges, and the bars extending from the boxes indicate the highest and lowest values $\left(n=7,{ }^{*} P<0.05\right)$. All data are taken from OVX mice. Left panels represent day- 3 immunostaining, illustrating increased cell numbers positive for TNF- $\alpha$ in the wild-type OVX wounds. Scale bars represent $50 \mu \mathrm{m}$.
\end{abstract}

been documented (23), indicating that the decreased accumulation of leukocytes in the wounds of the OVX $M I F$ null mice could not be attributed to systemic differences in hematopoiesis. Since TNF- $\alpha$ can induce MIF expression by macrophages and MIF in turn stimulates cellular TNF- $\alpha$ production (17), we reasoned that MIF regulation of the wound inflammatory response was linked to local TNF- $\alpha$ expression. To determine whether an absence of endogenous MIF resulted in a dampened TNF- $\alpha$ response in OVX mice in vivo, we compared tissue levels of TNF- $\alpha$ during the course of wound healing. In the wild-type OVX mice, reduced estrogen resulted in increased TNF- $\alpha$ protein levels from days 3-14 after wounding (Figure $4 \mathrm{~d}$ ). Intriguingly, after OVX in the animals null for MIF, wound TNF- $\alpha$ levels were reduced as compared with wild-type OVX wounds, suggesting that MIF is a key regulatory component necessary for in vivo wound TNF- $\alpha$ expression.

Reversal of impaired healing in OVX mice by anti-MIF antibodies. The lack of an impaired response to wounding in the OVX MIF null animals raised the possibility that the null animals were unable to mount a wounding response of greater magnitude than that observed in all the studies, regardless of hormonal status. In order to address this, MIF null intact mice were treated with exogenous agents, and the healing response was deter- mined. Both TGF- $\beta 1$ and rhMIF applied at the time of wounding stimulated marked inflammation and prolonged the healing time, suggesting that the MIF null mice were able to mount a wounding response, in terms of delayed healing and excessive inflammation, under specific circumstances but not in the absence of estrogen (Figure $5 \mathrm{a}$ ). In addition, not only could exogenous rhMIF delay healing, suggesting that supraphysiological doses of MIF were detrimental to normal wound repair (Figure 5a), but specific doses of antiMIF antibodies injected intradermally immediately before wounding in OVX wild-type mice reversed the delayed-healing phenotype, implicating MIF as a crucial component of impaired cutaneous repair in the absence of estrogen (Figure 5b).

Estrogen directly modulates macrophage MIF expression through the ER. The observation that the impaired woundhealing phenotype, resulting from an absence of estrogen, was absent in the MIF null mice, consistent with a dampened inflammatory response, suggested that estrogen directly or indirectly modulated inflammatory cellderived MIF expression. In concordance with the in vivo upregulation of MIF protein and mRNA expression in wound tissue of OVX mice (Figure 2), we showed that estrogen directly inhibited MIF production by activated murine macrophages (and by activated human 
monocytes) (data not shown) in a dose-dependent manner (Figure 6a). In all experiments, an estrogen concentration of $10^{-8} \mathrm{M}$ had the most pronounced effect; however, estrogen up to $10^{-11} \mathrm{M}$ had a significant effect on MIF production in vitro. Estrogen acting through its receptor regulates cell growth, differentiation, and gene expression (32). The recognition of at least two isoforms of ER, ER- $\alpha$ and ER- $\beta$, has increased our understanding of the actions of estrogen. Recent results have clearly implicated the importance of both ER- $\alpha$ and ER- $\beta$ in tissue-specific activities, resulting from genomic and rapid nongenomic mechanisms (32). To document a specific role for the ER in MIF modulation, we determined the expression of ER- $\alpha$ and ER- $\beta$ receptors in $\mathrm{CD} 14^{+}$human monocytes. Intracellular staining for both receptor isoforms was apparent (Figure 6b), implicating both ER- $\alpha$ and ER- $\beta$ as potential mediators of estrogen action on inflammatory cells. With the use of murine macrophages from wild-type and ER- $\alpha$ null mice, in the absence of ER- $\alpha$, LPS induced an increase in MIF production; however, estrogen had no effect, implicating this receptor as an integral component required for MIF downregulation (Figure 6c).

Transfection of ER-positive cells (MCF7 cells, which predominantly express ER- $\alpha$ ) with a MIF promoterluciferase reporter construct confirmed the inhibitory action of estrogen on LPS-induced promoter activity (Figure 6d). The effects of estrogen, either genomically or nongenomically through interaction with secondmessenger systems, occur at the level of transcription. Moreover, the ER antagonist ICI 182,780 reversed the inhibition of MIF promoter activity by estrogen (Figure 6e). Agonist activity at the ER- $\alpha$ with PPT (30) inhibited LPS-induced promoter activity to the same degree as ligand treatment with $10^{-8} \mathrm{M}$ estrogen. HPTE (29), which can act as both an ER- $\alpha$ agonist and an ER- $\beta$ antagonist depending on the relative ratios of the ER isoforms, with predominant ER- $\alpha$ agonist action when both isoforms are expressed, inhibited LPSinduced MIF expression in MCF7 cells in the absence of estrogen (ER- $\alpha$ agonist action). These data confirm the requirement of ER for MIF downregulation and suggest that the ER- $\alpha$ isoform is involved in estrogenregulated MIF inhibition.

\section{Discussion}

By gene targeting, we have documented a pivotal role for MIF in cutaneous wound healing. Although MIF has been shown in numerous studies to function as a proinflammatory agent in pathophysiological processes, our data are the first to implicate a major role for this protein in the cutaneous wound-healing response. Here, we demonstrate that MIF is expressed in a distinct spatial and temporal profile during the murine wound-healing response, localizing to keratinocytes and macrophages. Previous studies have highlighted the importance of

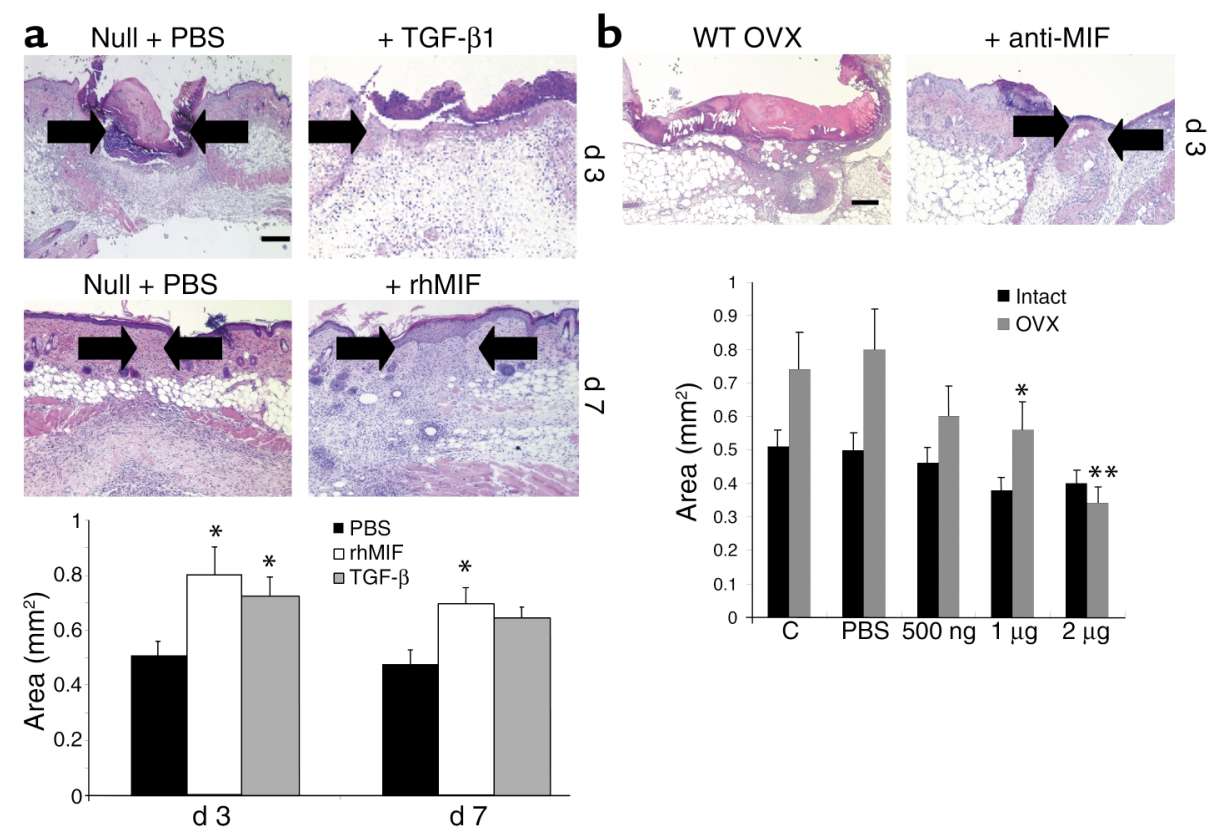

Figure 5

Treatment of MIF null wounds with TGF- $\beta$ (200 ng per wound) results in delayed healing as compared with vehicle-treated wounds (a, top panels). Wounds are representative of day 3 after wounding. Arrows demarcate wound edges. Treatment of MIF null wounds with rhMIF results in impaired healing (a, bottom panels) (day 7 after wounding, $1-\mu \mathrm{g}$ dose) as compared with vehicle (PBS). Scale bar represents 100 $\mu \mathrm{m}$. Results represent means \pm SEM $\left(n=5,{ }^{*} P<0.05\right)$. (b) Neutralization of MIF in wild-type OVX mice with anti-MIF antibodies leads to improved healing as compared with vehicle alone (day 3 after wounding). Arrows demarcate wound edges, which cannot be visualized in the OVX mice. Graph illustrates wound areas at day 3 after wounding in a dose-response study with increasing doses of anti-MIF antibodies injected at the time of wounding. Scale bar represents $100 \mu \mathrm{m}$. Results represent means $\pm \operatorname{SEM}\left(n=5,{ }^{*} P<0.05,{ }^{*} P<0.01\right)$. C, no injection at wound site; PBS, vehicle control. 
a
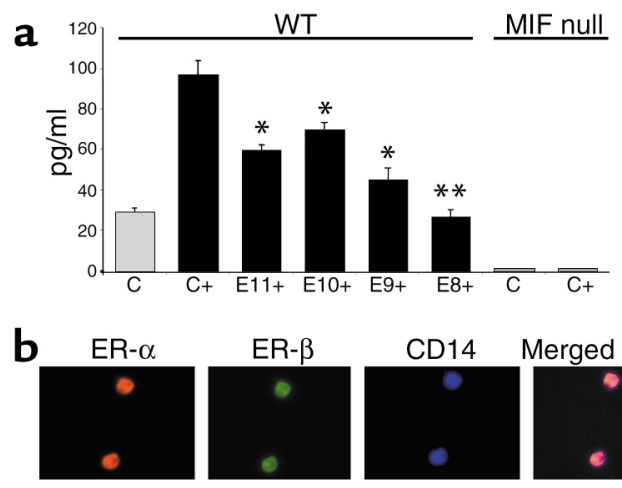

C

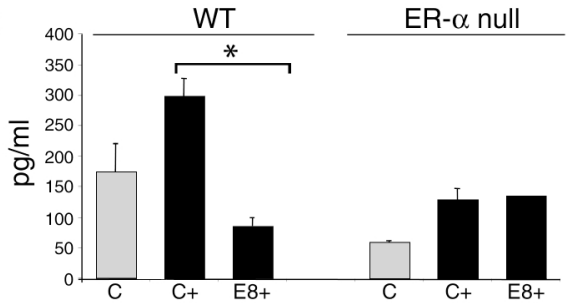

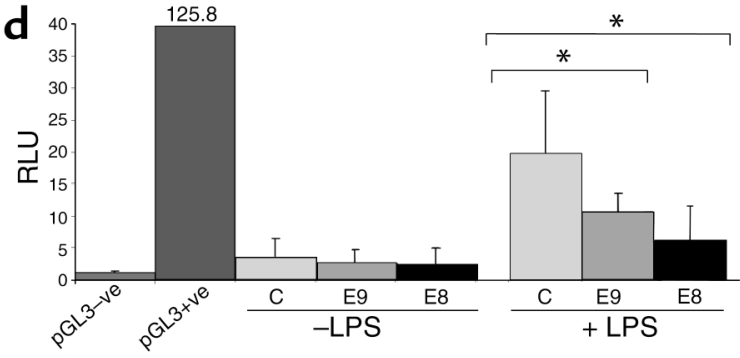

Merged image

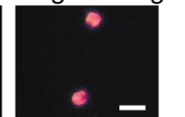

e 30

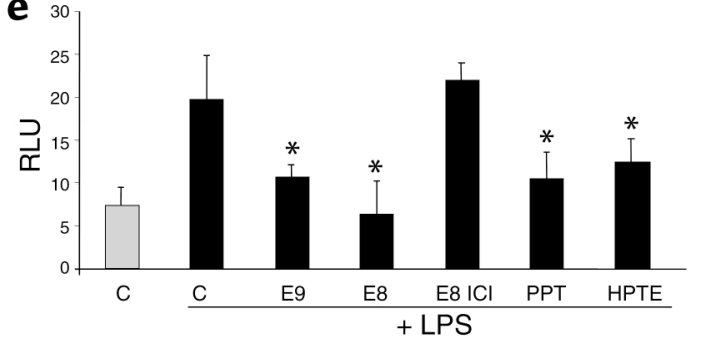

Figure 6

Murine macrophage MIF production, by LPS-activated cells, is decreased with concurrent estrogen treatment (a). Results represent means $\pm \operatorname{SEM}\left(n=10\right.$ for each group) ${ }^{*} P<0.05,{ }^{*} P<0.01$, all compared with control media plus LPS-activated macrophages $(C+)$. LPS activation significantly increased MIF production as compared with control media alone $(P<0.05)$. (b) Immunostaining illustrates that monocytes (CD14+ cells) express both ER- $\alpha$ and ER- $\beta$ isoforms. The far right panel of $\mathbf{b}$ represents a merged image of all three panels (ER- $\alpha$, ER- $\beta$, and CD14). Scale bar represents $10 \mu \mathrm{m}$. (c) LPS treatment increases MIF levels from wild-type murine cells and is inhibited by estrogen. In the absence of ER- $\alpha$ (ER- $\alpha$ null), estrogen fails to downregulate LPS-induced MIF production. LPS significantly increased MIF in both wild-type and ER- $\alpha$ null macrophages $(P<0.05)$. Seeding density of cells is three times higher than in Figure 5 a. Results represent means \pm SEM ( $n=5$ for each group, ${ }^{*} P<0.05$ as compared with LPS-activated cells). (d) MCF7 ER-expressing cells transfected with a MIF promoter-luciferase reporter construct showed increased activity after LPS activation (a). Estrogen (10 $\left.0^{-8} \mathrm{M}\right)$ inhibited this activity. Transfection with $\mathrm{pGL3}$ basic plasmid acted as a negative control ( $\mathrm{PGL3}-\mathrm{ve}$ ), and pGL3 control plasmid as a positive control (pGL3+ve). Results represent means \pm SEM $\left(n=3-5\right.$ for each group, ${ }^{*} P<0.05$ as compared with LPS-activated cells). (e) MCF7 ER-expressing cells transfected with a MIF promoter-luciferase reporter construct showed inhibition of LPS-activated MIF production by estrogen and estrogen agonists (PPT, HPTE) and reversal of estrogenic effects by ER antagonist (ICI). Results represent means \pm SEM for three experiments ( ${ }^{*} P<0.05$ as compared with LPS-activated cells). C, control media alone;,++ LPS treatment for

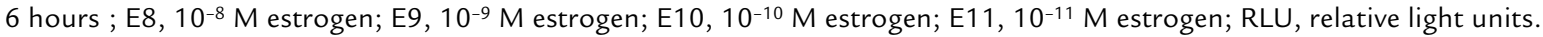

estrogen in maintaining a rapid rate of healing in both animal and human models, suggesting that the marked reduction of estrogen with age in both females and males plays a major role in age-related impaired woundhealing states (2-8). Moreover, evidence suggests that a crucial mechanism underlying the responses to estrogen involves a dampening of the inflammatory response, resulting in reduced proteolytic activity and enhanced matrix deposition (6). Upregulation of MIF expression was observed in wound tissue from OVX animals as compared with intact controls. In the absence of MIF (MIF null mice), estrogen depletion had no adverse effect on wound healing. Moreover, in wild-type mice in the absence of estrogen, MIF inactivation using exogenous topical anti-MIF antibodies reverses the delay in healing and the exaggerated and prolonged inflammatory response. Importantly, no significant differences in the rate of healing were observed between intact wild-type and MIF null mice, suggesting that in the presence of estrogen the absence of MIF has no effect. Taken together, these data implicate MIF as the integral component of estrogen-mediated responses, since in the absence of endogenous MIF normal physiological wound healing occurs despite reduced systemic estrogen.

In concordance with previous studies implicating MIF as a mediator of pathological disease processes, including glomerulonephritis, endotoxic shock, and the cutaneous delayed-type hypersensitivity reaction $(14,18,22)$, a key mechanism by which MIF appears to participate in the pathogenesis of impaired wound healing involves a marked increase in the inflammatory response, as observed in wild-type intact mice treated exogenously with rhMIF. The exaggerated inflammatory response in the OVX wounds of wild-type animals was associated with enhanced expression of tissue TNF- $\alpha$, which was curtailed in the MIF null mice. Moreover, topical neutralization of MIF in wild-type OVX mice results in a reversal of the impaired healing response and inflammation. These data suggest that MIF is a crucial mediator of excessive inflammation in the absence of estrogen. Previous studies have documented the temporally regulated upregulation of TNF- $\alpha$ in the wound-healing process, expressed by both inflammatory cells and keratinocytes (33). In vitro, it has been shown that MIF 
induces macrophage-derived TNF- $\alpha$ production. Moreover, various proinflammatory mediators, including LPS and TNF- $\alpha$ itself, are potent inducers of MIF secretion, providing a feedback loop that is disrupted in the MIF null mice leading to a dampening of inflammatory cell influx. In systemic inflammation $(E$. coli peritonitis), anti-MIF antibodies protect TNF- $\alpha$ null mice from death, suggesting that TNF- $\alpha$ is not the major target of MIF-mediated effects in sepsis (34). Future studies using such null mouse models rendered hypogonadal would shed light on the specific interactions between MIF and TNF- $\alpha$.

A previously unrecognized link between MIF and estrogen has emerged from this study. Although MIF has been implicated in a variety of reproductive processes and is expressed in a number of hormonally responsive tissues, no direct association between MIF and estrogen has been considered $(11,12)$. LPS-induced MIF production by macrophages is inhibited by estrogen in a dose-dependent fashion. Moreover, in vivo expression of MIF during wound healing is downregulated in the presence of estrogen, supporting the hypothesis that MIF is directly modulated by the hormonal milieu. The importance of MIF in this process was confirmed in studies investigating the wound-healing response of animals null for the MIF gene. Whereas reduced systemic estrogen in wild-type (OVX) mice results in a markedly impaired rate of wound healing associated with excessive inflammation, in MIF null mice the impaired-healing phenotype is not observed. This appears to be a direct result of reduced estrogen levels, since MIF null mice can mount a prolonged wound-healing response with increased inflammation under specific circumstances. These data implicate MIF as a necessary intermediate required for uncontrolled inflammation and impaired healing in the absence of estrogen, a hypothesis confirmed by the observed improved healing when hypogonadal wild-type mice are treated with anti-MIF antibodies at the time of wounding. Intact MIF null and wild-type animals heal similarly, suggesting that only in the absence of estrogen are MIF levels pathologically increased, leading to unchecked inflammation and impaired healing. Our data reaffirm the concept that in the absence of infection, the inflammatory response in the absence of estrogen is excessive and detrimental to cutaneous wound healing, and MIF is an important regulator of such a response $(1,2,35)$.

The downregulation of MIF appears to form an integral component of the anti-inflammatory effects of estrogen in vivo, a pathway that may be applicable to other pathophysiological processes involving estrogen, such as atherosclerosis, restenosis injury, and osteoporosis. Moreover, these data correlate with the sexual dimorphism observed in the response to sepsis and trauma hemorrhage. Females are reported to have an improved outcome in these situations, and estrogen replacement in animal models is considered as a possible adjunct for prevention of sepsis $(36,37)$. Wound healing involves a local inflammatory response that distin- guishes it pathophysiologically from sepsis, in which raised systemic MIF levels have been observed. Importantly, no differences in MIF expression in normal skin, nor serum MIF levels, were observed between the intact and OVX wild-type mice. Estrogen appears to markedly downregulate MIF expression by LPS-activated murine macrophages and has no effect on basal (unactivated) expression (G.S. Ashcroft, unpublished observation) or on basal levels of MIF in MCF7 cells transfected with the MIF-luciferase construct. It is therefore not surprising that basal tissue (normal skin) and serum levels are similar regardless of the level of systemic or local estrogen. However, after local tissue injury, recruitment and subsequent activation of inflammatory cells at the wound site results in increased expression of MIF locally. In the absence of estrogen, this local increase in MIF expression occurs unchecked, resulting in excessive inflammation and delayed healing.

The existence of a protein responsible for specific binding of estradiol in the uterus was recognized over 40 years ago, and in 1996 a novel ER, termed ER- $\beta$ to distinguish it from the original ER- $\alpha$, was cloned. Recent studies have highlighted the tissue-specific expression of such receptors and the regulation of immune function in vivo dependent on the presence of the ER (38-40). However, estrogen may also act through nonclassic membrane receptors, leading to rapid intracellular responses (41). We have further investigated the mechanisms underlying estrogen inhibition of MIF expression, and our data implicate the ER in this process. Notably, both receptors colocalize in monocytes, consistent with the coexpression of ER$\alpha$ and ER- $\beta$ in other cells, such as rat cardiac fibroblasts, and the formation of heterodimers in vitro (40, 42), suggesting that specific actions of estrogen are potentially mediated by one or both ER isoforms. Our data confirm the inhibition of LPS-induced MIF promoter activity in the presence of estrogen but also implicate the ER in the regulation of MIF by estrogen, since estrogen fails to dampen MIF expression in transfected ER-expressing MCF7 cells in the presence of an ER antagonist (ICI 182,780) and agonist activity (PPT, HPTE) at the ER- $\alpha$ inhibits MIF expression. These findings implicate the ER as a therapeutic target for manipulation of MIF levels locally in wound repair and in other pathological conditions associated with MIF expression and a destructive inflammatory response. Impaired wound healing in the elderly poses an immense problem in terms of cost, morbidity, and mortality. We have previously reported that exogenous estrogen accelerates healing in both elderly women and men, suggesting that the systemic decline in estrogen levels in women, the reduced production of estrogenic precursors such as adrenal DHEA in men and women, and the altered local aromatization of systemic precursors to local estrogen influence healing in both sexes. We suggest that a pivotal mechanism whereby estrogen modulates the local wound-healing response and downregulates inflammation is through ER-mediated inhibition 
of MIF. Pharmacological agonism of, for example, ER- $\alpha$ in macrophages may be an intriguing approach to downregulate MIF expression. In addition, we suggest that local MIF inhibition is a potentially focused approach to therapeutically accelerate cutaneous healing in impaired age-related wound-healing states.

\section{Acknowledgments}

G.S. Ashcroft is a recipient of a Wellcome Trust Senior Fellowship in Clinical Science. The ER- $\beta$ antibody was kindly supplied by Phillipa Saunders (MRC Reproduction Unit, Edinburgh, United Kingdom). The MCF7 cells were supplied by Neil Anderson (University of Manchester, Manchester, United Kingdom). The antirat MIF antibody was supplied by Jun Nishihira (Hakkaido University Graduate School of Medicine, Sapporo, Japan). We are grateful for the technical assistance of Yvonne Davidson.

1. Ashcroft, G.S., et al. 2000. Secretory leukocyte protease inhibitor (SLPI) mediates non-redundant functions necessary for normal wound healing. Nat. Med. 6:1147-1153.

2. Ashcroft, G.S., Dodsworth, J., Boxtel, E., Horan, M., and Ferguson, M.W.J. 1997. Estrogen accelerates cutaneous wound healing associated with an increase in TGF- $\beta 1$ levels. Nat. Med. 3:1209-1215.

3. Calvin, M., Dyson, M., Rymer, J., and Young, S.R. 1998. The effects of ovarian hormone deficiency on wound contraction in a rat model. $\mathrm{Br}$. J. Obstet. Gynaecol. 105:223-227.

4. Jorgensen, O., and Schmidt, A. 1962. Influence of sex hormones on granulation tissue formation and on healing of linear wounds. Acta Chir. Scand. 124:1-10.

5. Pallin, B., Ahonen, J., and Zederfeldt, B. 1975. Granulation tissue formation in oophorectomized rats treated with female sex hormones: II. Acta Chir. Scand. 141:710-714.

6. Ashcroft, G.S., Greenwell-Wild, T., Horan, M., Wahl, S.M., and Ferguson, M. 1999. Topical estrogen accelerates cutaneous wound healing in aged humans associated with an altered inflammatory response. Am.J. Pathol. 155:1137-1146.

7. Pirila, E., et al. 2002. Chemically modified tetracycline (CMT-8) and estrogen promote wound healing in ovariectomized rats: effects on matrix metalloproteinase-2, membrane type 1 matrix metalloproteinase, and laminin-5 $\gamma 2$-chain. Wound Repair Regen. 10:38-51.

8. Pirila, E., et al. 2001. Wound healing in ovariectomized rats: effects of chemically modified tetracycline (CMT-8) and estrogen on matrix metalloproteinases $-8,-13$ and type I collagen expression. Curr. Med. Chem. 8:281-294.

9. Margolis, D.J., Knauss, J., and Bilker, W. 2002. Hormone replacement therapy and prevention of pressure ulcers and venous leg ulcers. Lancet. 23:675-677.

10. Berard, A., Kahn, S.R., and Abenhaim, L. 2001. Is hormone replacement therapy protective for venous ulcer of the lower limbs? Pharmacoepidemiol. Drug Saf. 10:245-251.

11. Arcuri, F., et al. 2001. Migration inhibitory factor in the human endometrium: expression and localization during the menstrual cycle and early pregnancy. Biol. Reprod. 64:1200-1205.

12. Wada, S., et al. 1999. Induction of macrophage migration inhibitory factor in human ovary by human chorionic gonadotrophin. Hum. Reprod. 14:395-399.

13. Froidevaux, C., Roger, T., Martin, C., Glauser, M., and Calandra, T. 2001. Macrophage migration inhibitory factor and innate immune responses to bacterial infections. Crit. Care Med. 29:S13.

14. Bloom, B.R., and Bennett, B. 1966. Mechanism of a reaction in vitro associated with delayed-type hypersensitivity. Science. 153:80-82.

15. Nishihira, J., Koyama, Y., and Mizue, Y. 1998. Identification of macrophage migration inhibitory factor (MIF) in human vascular endothelial cells and its induction by lipopolysaccharide. Cytokine. 10:199-205.

16. Abe, R., Shimizu, T., Ohkawara, A., and Nishihira, J. 2000. Enhancement of macrophage migration inhibitory factor (MIF) expression in injured epidermis and cultured fibroblasts. Biochim. Biophys. Acta. 1500:1-9.

17. Nathan, C.F., Karnovsky, M., and David, J.R. 1971. Alterations of macrophage functions by mediators from lymphocytes. J. Exp. Med. 133:1356-1376

18. Bernhagen, J.T., et al. 1993. MIF is a pituitary-derived cytokine that potentiates lethal endotoxaemia. Nature. 365:756-759.

19. Calandra, T., and Bucala, R. 1995. Macrophage migration inhibitory factor: a counter-regulator of glucocorticoid action and critical mediator of septic shock. J. Inflamm. 47:39-51.

20. Calandra, T., Bernhagen, J., Mitchell, R., and Bucala, R. 1994. The macrophage is an important and previously unrecognized source of macrophage migration inhibitory factor. J. Exp. Med. 179:1895-1902.

21. Santos, L., Hall, P., Metz, C., Bucala, R., and Morand, E. 2001. Role of macrophage migration inhibitory factor (MIF) in murine antigen-induced arthritis: interaction with glucocorticoids. Clin. Exp. Immunol. 123:309-314.

22. Yang, N., et al. 1998. Reversal of established rat crescentic glomerulonephritis by blockade of macrophage migration inhibitory factor (MIF): potential role of MIF in regulating glucocorticoid production. Mol. Med. 4:413-424.

23. Honma, N., et al. 2000. Deficiency of the macrophage migration inhibitory factor gene has no significant effect on endotoxaemia. Immunology. 100:84-90.

24. Yang, X., et al. 1999. Targeted disruption of SMAD3 results in impaired mucosal immunity and diminished $\mathrm{T}$ cell responsiveness to TGF- $\beta$. EMBO J. 18:1280-1291.

25. Kitaichi, N., et al. 2000. Inhibition of experimental autoimmune uveoretinitis with anti-macrophage migration inhibitory factor antibodies. J. Curr. Eye Res. 20:109-114.

26. Saunders, P.T.K., et al. 2001. Differential expression of oestrogen receptor a and $\beta$ proteins in the testes and male reproductive tract of human and non-human primates. Mol. Human Reprod. 7:227-236.

27. Ashcroft, G.S., et al. 1997. Age-related differences in the temporal and spatial regulation of matrix metalloproteinases (MMPs) in normal skin and acute cutaneous wounds of healthy humans. Cell Tissue Res. 290:581-591.

28. Kraichely, D.M., Sun, J., Katzenellenbogen, J., and Katzenellenbogen, B. 2000. Conformational changes and coactivator recruitment by novel ligands for estrogen receptor- $\alpha$ and estrogen receptor- $\beta$ : correlation with biological character and distinct differences among SRC coactivator family members. Endocrinology. 141:3534-3545.

29. Gaido, K.W., et al. 1999. Differential interaction of the methoxychlor metabolite 2,2-bis-(p-hydroxyphenyl)-1,1,1-trichloroethane with estrogen receptors $\alpha$ and $\beta$. Endocrinology. 140:5764-5753.

30. Stauffer, S.R., et al. 2000. Pyrazole ligands: structure-affinity/activity relationships and estrogen receptor-a-selective agonists. J. Med. Chem. 43:4934-4947.

31. Shimizu, T., Abe, R., Ohkaear, A., Mizue, Y., and Nishihira, J. 1997. Macrophage migration inhibitory factor is an essential immunoregulatory cytokine in atopic dermatitis. Biochem. Biophys. Res. Commun. 240:173-178.

32. Fankelstein, E., et al. 2000. Multiple actions of steroid hormones - a focus on rapid, non-genomic effects. Pharm. Rev. 52:513-555.

33. Hubner, G., et al. 1996. Differential regulation of pro-inflammatory cytokines during wound healing in normal and glucocorticoid-treated mice. Cytokine. 8:548-556.

34. Calandra, T., et al. 2000. Protection from septic shock by neutralization of macrophage migration inhibitory factor. Nat. Med. 6:164-170.

35. Ashcroft, G.S., et al. 1999. Mice lacking Smad3 show accelerated wound healing and an impaired local inflammatory response. Nat. Cell Biol. 1:260-266.

36. Knoferl, M.W., et al. 2002. Female sex hormones regulate macrophage function after trauma-hemorrhage and prevent increased death rate from subsequent sepsis. Ann. Surg. 235:105-112.

37. Merkel, S.M., Alexander, S., Zufall, E., Oliver, J., and Huet-Hudson, Y. 2001. Essential role for estrogen in protection against Vibrio vulnificus-induced endotoxic shock. Infect. Immun. 69:6119-6122.

38. Couse, J.F., Lindzey, J., Grandien, K., Gustafsson, J., and Korach, K. 1997. Tissue distribution and quantitative analysis of estrogen receptor-a (ERa) and estrogen receptor- $\beta(\operatorname{ER} \beta)$ messenger ribonucleic acid in the wild-type and ERa-knockout mouse. Endocrinology. 138:4613-4621.

39. Grohe, C., et al. 1997. Cardiac myocytes and fibroblasts contain functional estrogen receptors. FEBS Lett. 13:107-112.

40. Erlandsson, M.C., Ohlsson, C., Gustafsson, J., and Carlstein, H. 2001. Role of estrogen receptors $\alpha$ and $\beta$ in immune organ development and in oestrogen-mediated effects on thymus. Immunology. 103:17-25.

41. Ropero, A.B., Soria, B., and Nadal, A. 2002. A nonclassical estrogen membrane receptor triggers rapid differential actions in the endocrine pancreas. Mol. Endocrinol. 3:497-505.

42. Cowley, S.M., Hoare, S., Mosselman, S., and Parker, M. 1997. Estrogen receptors $\alpha$ and $\beta$ form heterodimers on DNA. J. Biol. Chem. 272:19858-19862. 